\title{
Abundancia, riqueza y diversidad de insectos asociados al cultivo de Marango (Moringa oleifera L.) en Managua, Nicaragua
}

\section{Abundance, richness and diversity of insects associated to cultivation of Marango (Moringa oleífera L.) in Managua, Nicaragua}

\author{
Edgardo Jiménez-Martínez ${ }^{1}$, Víctor Jirón Cortez², Maritza Téllez Manzanarez² \\ ${ }^{1} \mathrm{PhD}$. en Entomología, ${ }^{2}$ Graduados de la carrera de Ingeniería Agronómica, Universidad Nacional Agraria
}

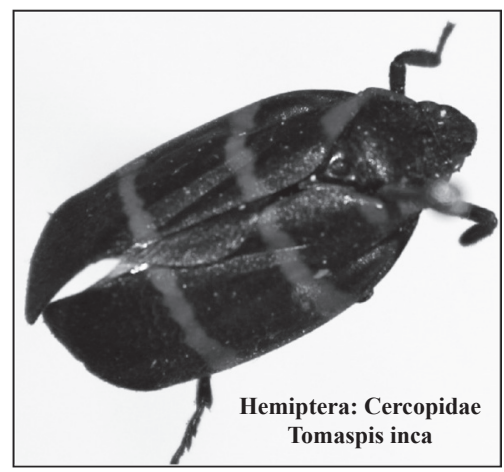

\section{RESUMEN}

El marango (Moringa oleifera L.) es un árbol de la familia Moringácea que crece en el trópico, es un cultivo de importancia en Centro América como en el resto de América Latina. En Nicaragua durante los últimos años ha crecido el interés por este cultivo por su potencial nutritivo en la alimentación de ganado bovino, porcino y aves. Sin embargo, el marango como cualquier otro cultivo se ha visto afectado por muchos problemas, entre ellos algunos de tipo fitosanitarios, causando éstos una drástica reducción en los rendimientos totales de este rubro. Con el objetivo de contribuir en el aporte de información para el manejo de estos insectos, se realizó este estudio para identificar los principales insectos asociados al cultivo de marango, conocer la variación temporal de la población de insectos, en un período comprendido de seis meses, conocer la abundancia, riqueza y su comportamiento. Este trabajo se realizó en el período comprendido de noviembre de 2012 a abril del 2013 en Managua, en dos fincas, Las Mercedes, de la Universidad Nacional Agraria y la finca del Centro Nacional de Investigación Agropecuaria, perteneciente al Intituto Nicaragüense de Tecnología Agropecuaria. En ambas fincas se utilizaron trampas para la captura de insectos, estas fueron 12 trampas de caída libre (Pitfall traps) y 12 trampas de recipientes de plástico de un galón con agua y melaza. El total de trampas ubicadas por finca fue de 24 . Los muestreos se realizaron semanalmente y los insectos colectados en el campo fueron llevados al laboratorio para su respectiva identificación. De acuerdo a lo resultados obtenidos en el estudio, los principales ordenes de insectos encontrados fueron Orthóptera, Mantodea, Hemíptera, Dermáptera, Homóptera, Thysanoptera, Coleóptera, Díptera, Lepidóptera, Hymenóptera, Blattodea, Isóptera y Megalóptera, se encontró que la mayor abundancia de insectos fue en la finca Las Mercedes y la mayor riqueza de insectos en la finca del INTA. El índice de diversidad fue mayor en la finca del INTA que en Las Mercedes con índices de 1.14 y 1.13 respectivamente. Este es el primer estudio en Nicaragua donde se identifican y describen los principales insectos asociados al cultivo del marango. Palabras clave: plagas, moringácea, fluctuación poblacional.

\section{ABSTRACT}

The marango (Moringa oleiffera L.) is a tree of the family Moringácea that grows in the tropics, it is a crop of great importance in both Latin América and also in Central América. In Nicaragua, during the past few years, has grown the interest in this crop majorly because of its nutritional potential in the supply of cattle, pigs and birds. However, the marango as any other crop is been affected by many problems, including some phytosanitary problems, causing these, a drastic reduction in the total returns of this crop. With the aim of contributing to the information for the management of insect pests in Marango, this study was conducted in order to identify the principal insects associated to marango, in additión, to know the population fluctuations of these insects in the different times of the year, to learn about the insect abundance, richness and their behavior. This work was carried out in the period from November 2012 to April 2013 in Managua, in two farms, Las Mercedes and the national center for research in agriculture, belongs to the National Institute for Agriculture Technology (INTA). In both farms, traps were placed for the capture of insects; these were 12 Pit fall trap and 12 traps of plastic gallon containers with water and molasses. The total number of traps placed per farm was 24 . The sampling was done weekly and insects collected in the field were brought to the laboratory for identificatión. According to the results obtained in the study, the main orders of insects found associated to marango were Orthóptera, Mantodea, Hemíptera, Dermáptera, Homóptera, Thysanoptera, Coleóptera, Díptera, Lepidoptera, Hymenoptera, Blattodea, Isóptera and Megaloptera. It was found that the greater insect abundance was found in the farm Las Mercedes, and the greater insect richness was found on the farm INTA. The diversity index was higher in the INTAb farm than in Las Mercedes farm with rates of 1.14 and 1.13 respectively. This is the first study in Nicaragua where it is identified and described the major insects associated to marango.

Keywords: Pest, moringacea, population fluctuation.

Recibido: 3 de noviembre 2014

Aceptado: 30 de abril 2015 
$\mathrm{E}$ 1 marango (Moringa oleifera L.) es un árbol de la familia Moringácea que crece en el trópico y es una especie originaria del sur de Himalaya, noreste de la India, Bangladesh, Afganistán y Pakistán (Rajangam et al., 2011), (Makkar y Becker, 1997). En América Latina se introdujo y naturalizó en 1920 como un árbol ornamental y fue utilizado como cerca viva y cortinas rompe vientos (Nikolaus et al., 1998). El marango, crece en cualquier tipo de suelo, por lo que se recomienda la siembra para la alimentación humana y animal. Hoy en día organizaciones humanitarias del mundo lo recomiendan como alimento para combatir la hambruna (D'Souza, y Kulkarni, 1990). Según FAO (2003) sus hojas poseen grandes cualidades nutritivas, con un alto contenido proteico de $27 \%$ (tanto como el huevo y el doble de la leche), con cantidades significativas de calcio (cuatro veces superior a lo contenido en la leche); contiene además, hierro, fósforo y potasio (tres veces más que el banano), vitamina $\mathrm{A}$ (cuatro veces más que la zanahoria) y vitamina C (30 veces más que la naranja), la lista de beneficios del árbol de la vida (marango), no concluye con estos impresionantes datos.

Es una de las especies vegetales con mayor contenido de aceite (35\%). La madera de este árbol sirve como leña y para hacer carbón, además de celulosa para producir papel de alta calidad. La planta es buena purificadora de agua y al utilizarse como forraje, se destaca otra larga lista de características benéficas, ya que sirve tanto para ganado vacuno como porcino, ovino, caprino y avícola, entre otros, a los que aporta importantes incrementos en el rendimiento, aumento de peso y producción de leche (Castellón y González 1996.). El marango es un árbol de crecimiento rápido, alcanza una altura de siete a 12 metros hasta la corona, su tronco posee un diámetro de 20 a $30 \mathrm{~cm}$, tiende a desarrollar raíces fuertes y profundas (Johnson, 2005) (UNA, 2004), y tiene una vida relativamente corta, alcanzando un promedio de 20 años. Posee hojas compuestas alternas imparipinnadas con una longitud total de $30 \mathrm{a} 70 \mathrm{~cm}$. Las flores son blancas, cremosas, con estambres amarillos y nacen en racimos. El fruto es una cápsula colgante color castaño, triangular, con $30 \mathrm{~cm}$ de largo y $1.8 \mathrm{~cm}$ de diámetro. Las semillas son de color castaño oscuro con tres alas blancas delgadas. La raíz es principalmente gruesa. El árbol florece y produce semillas durante todo el año (Johnson, 2005).

Por ser una planta de origen tropical, se desarrolla en climas semi-áridos, semi-húmedos y húmedos. El marango crece bien en alturas que van desde el nivel del mar hasta los 1200 metros de altitud y prospera en temperaturas altas, considerándose óptimas para un buen comportamiento las que están entre 24 y $32^{\circ} \mathrm{C}$ (UNA, 2004). El agua afecta el crecimiento y desarrollo de las plantas ya que actúa como constituyente, solvente, responsable de la turgencia celular y reguladora de su temperatura, por lo que la cantidad, frecuencia e intensidad de las lluvias determinan en gran medida la adaptación de una especie forrajera particular a un ambiente determinado. El marango necesita al menos $700 \mathrm{~mm}$ anuales, aunque hay reportes de lugares del pacífico de Nicaragua donde con $300 \mathrm{~mm}$ crece muy bien. Se ha observado un buen comportamiento en lugares con precipitaciones anuales de $2000 \mathrm{~mm}$ (UNA, 2004).

En Nicaragua, el marango constituye una alternativa alimenticia forrajera para el ganado bovino, porcino y alimentación de aves de corral, y es empleado para mejorar su producción sobre todo en los meses secos o de escases de alimento forrajero. El marango como cualquier otro cultivo se ha visto afectado por muchos problemas, entre ellos algunos de tipo fitosanitarios, causando estos una drástica reducción en los rendimientos totales de este rubro.

Se mencionan tres plagas insectiles importantes tales como los comejenes, las hormigas y los insectos chupadores como mosca blanca y chinche (Martínez et al., 2011). Con el objetivo de contribuir en el aporte de información para el manejo de estas plagas se realizó este estudio para identificar los principales insectos asociados al cultivo del marango, su fluctuación poblacional y enemigos naturales, que permita la implementación de un plan de manejo integrado a corto, mediano y largo plazo, que ayude a los productores a reducir los daños ocasionados en plantaciones comerciales de marango en Nicaragua.

\section{MATERIALES Y MÉTODOS}

El estudio se realizó entre los meses de noviembre del 2012 a abril del 2013 en dos fincas experimentales donde se encuentran establecidas parcelas con el cultivo de marango. La primera es la hacienda Las Mercedes, propiedad de la Universidad Nacional Agraria (UNA), ubicada en Managua en el km 11 de la Carretera Norte, 300 metros al sur. La segunda es el Centro Nacional de Investigación Agropecuaria del Intituto Nicaraguense de Tecnología Agropecuaria (CNIA-INTA), ubicada también en Managua en el km 14 de la Carretera Norte, $2.5 \mathrm{~km}$ hacia el sur.

En Las Mercedes hay cuatro parcelas con un área de 0.25 hay de siete años de edad. El manejo consiste en corte de ramas y hojas cada 45 días, que se utiliza para la alimentación del ganado vacuno. En la finca CNIA-INTA, existen cuatro parcelas de marango de 2.5 , hay con edad de 10 años y cuyo objetivo es la extracción, una vez al año, de estacones o material vegetativo para la propagación del cultivo.

Diseño experimental. Consistió en la comparación de cuatro parcelas por finca. En cada parcela, tanto en Las Mercedes como en el CNIA-INTA se colocaron seis trampas de captura de insectos, tres trampas del tipo Pitfall Traps (trampas de caída libre) y tres trampas del tipo recipientes de plástico de un galón de capacidad con agua y melaza. El total de trampas por finca, fue de 12 del tipo caída libre y 12 del tipo galones con agua y melaza. 
Colección, identificación y descripción de los principales insectos. La colecta de los insectos se realizó semanalmente, utilizando dos tipos de trampas. El primer muestreo consistió en captura de insectos rastreros con trampas de caída libre (Pitfall-traps), y el segundo muestreo consistió en captura de insectos voladores y caminadores, utilizando trampas de galones plásticos con agua y melaza. Las muestras de los insectos fueron colectados en viales entomológicos, fueron rotulados con la fecha y el sitio de muestreo, posteriormente estos insectos fueron llevados al laboratorio de entomología de la UNA para ser montados e identificados.

Montaje e identificación de insectos. Se tomaron los insectos de los viales entomológicos y se colocaron en platos petrix de $14 \mathrm{~cm}$ de ancho y dos $\mathrm{cm}$ de largo para ser lavados en alcohol al 75\%, luego cada muestra de insectos se colocó en papel toalla para secar durante 30 minutos a temperatura ambiente, se contaron los insectos y se anotaron en una hoja de muestreo. Para el montaje en las cajas entomológicas, se procedió a la utilización de gradillas entomológicas, pinzas, pinceles, alfileres entomológicos número cuatro, marca MORPHO de 38 y $45 \mathrm{~mm}$ de longitud, poroplas con una medición de $59 \mathrm{~cm}$ de ancho y $121 \mathrm{~cm}$ de largo. Luego se ordenaron en las cajas entomológicas, cada insecto se rotuló con dos etiquetas, la primera etiqueta con información sobre fecha, finca, número de trampas, país, departamento y colector. La segunda etiqueta contenía el orden, familia, género y la especie a la que pertenece.

Los insectos fueron observados con el microscopio (color y forma) y se realizó una revisión bibliográfica para lograr una identificación final de los insectos hasta el nivel de familia. Luego fueron fotografiados para realizar verificaciones de especímenes con las especies existentes en el museo y también se consultaron literaturas y libros relacionados a la taxonomía de insectos. Además de la revisión de literatura específica, se realizó una verificación en el museo entomológico de la UNA.

Variables evaluadas. Las variables evaluadas fueron abundancia, riqueza e índice de diversidad, esta última según Shannon-Weaver, 1949.

Indice de diversidad de Shannon Weaver. El índice de ShannonWeaver se usa para medir la biodiversidad específica. Este índice se representa normalmente como H' y se expresa con un número positivo, que en la mayoría de los ecosistemas naturales varía entre 0 y 1 . No tiene límite superior o en todo caso lo da la base del logaritmo que se utilice. Los ecosistemas con mayores valores son los bosques tropicales y arrecifes de coral, y los menores las zonas desérticas La fórmula del índice de Shannon-Weaver es la siguiente:

$$
H^{\prime}=-\sum_{i=1}^{S} p_{i} \log _{2} p_{i} \frac{n_{i}}{N}
$$

Donde:

$S$ : Número de especies (la riqueza de especies)

$p_{i}$ : Proporción de individuos de la especie i respecto al total de individuos (es decir la abundancia relativa de la especie)

$\boldsymbol{n}_{\mathfrak{i}}$ : Número de individuos de la especie $i$

$N$ : Número de todos los individuos de todas las especies

De esta forma, el índice contempla la cantidad de especies presentes en el área de estudio (riqueza de especies), y la cantidad relativa de individuos de cada una de esas especies (abundancia).

Análisis de datos. Después de colectados los datos de insectos obtenidos en el campo, estos fueron arreglados por variables de familias de insectos por finca, luego cada variable fue comparada entre fincas, utilizando un análisis de comparación de t de student, usando el programa stadistical analysis system (SAS) (SAS, 2003). El nivel de significancia fue de $(p=0.05)$.

\section{RESULTADOS Y DISCUSIÓN}

En el cuadro 1 se presentan los principales órdenes, familias, géneros, especies, nombre común y hábito alimenticio de los insectos identificados según literatura de JiménezMartínez (2009), Nunes y Dávila, (2004) y Sáenz y De La Llana, (1990). Los ordenes registrados fueron Orthóptera, Hemíptera, Coleóptera, Díptera, e Hymenóptera.

\begin{tabular}{|c|c|c|c|c|}
\hline Orden & Familia & Género y especie & Nombre común & Hábito alimenticio \\
\hline \multirow[t]{4}{*}{ Orthóptera } & Gryllidae & Gryllus sp & Grillo & Masticador \\
\hline & Acrididae & Dephalella $S p$ & Chapulín & Masticador \\
\hline & Acrididae & Orphula sp & Chapulín & Masticador \\
\hline & Acrididae & Schistoserca nitens & Chapulín & Masticador \\
\hline \multirow[t]{3}{*}{ Hemiptera } & Cercopidae & Tomaspis cf & Salivazo & chupador \\
\hline & Cercopidae & Prosapia simulans & Salivazo & Chupador \\
\hline & Coreidae & Leptoglossus chilensis & Chinche & Chupador \\
\hline \multirow[t]{9}{*}{ Coleoptera } & Coccinellidae & Ylocorus cacti.VD & Malla & Masticador \\
\hline & Meloidae & & Pava & Masticador \\
\hline & Meloidae & Pyrota decorata & & Masticador \\
\hline & Crysomelidae & Hysonota alutacea & Tortuguilla & Masticador \\
\hline & Carabidae & Galerita $\mathrm{sp}$ & Escarabajo & Masticador \\
\hline & Carabidae & Conoderus sp & Escarabajo & Masticador \\
\hline & Carabidae & Pasimachus c.f. & Escarabajo & Masticador \\
\hline & Elateridae & & & Masticador \\
\hline & Elateridae & Conoderus sp & & Masticador \\
\hline Diptera & Tachinidae & & Mosca verde & Lamedor-chupador \\
\hline \multirow[t]{4}{*}{ Hymenóptera } & Vespidae & Polistes sp & Catala & Lamedor \\
\hline & Vespidae & & Catala & Lamedor \\
\hline & Apidae & Xylocopa $\mathrm{sp}$ & Abejorro & Lamedor \\
\hline & Apidae & Trigona silvestrianum & Congo & Lamedor \\
\hline
\end{tabular}


Abundancia. La abundancia total de insectos en las dos fincas fue de 6064 insectos, siendo la parcela de Las Mercedes la que presentó mayor número de insectos con 3328 , mientras que en la parcela del CNIA-INTA se regisraron 2736 insectos (figura, 1). Podemos decir que el tipo de manejo que se da en ambas fincas ha influido en la población de insectos. En la parcela de Las Mercedes, no se realiza ninguna aplicación para el manejo de plagas, únicamente se realiza deshierba cuando la maleza está muy desarrollada, y puede que por esta razón, haya mayor población de insectos. Además, hay un rebrote constante de hojas y brotes tiernos, lo que garantiza mejor calidad de alimentación a base de nitrógeno disponible para los insectos, mientras que en la parcela de marango del CNIA-INTA, los árboles son de mayor edad y el tipo de manejo que se da a ésta parcela es de corte para estacones para ser utilizados como material vegetativo de propagación, dos veces al año, en ésta parcela se hacían aplicaciones químicas para el control de insectos hasta antes de empezar el estudio, sobre todo para control de termitas y otros insectos. Las parcelas del CNIA-INTA, por ser una estación experimental, existe alrededor de la parcela de marango otras áreas de cultivos experimentales en los que se realizan aplicaciones de productos químicos para el control de insectos plagas, esto influye negativamente en

las poblaciones generales de insectos en el marango, por lo que ésta podría ser una razón que justifica la menor presencia de insectos en la parcela del CNIA-INTA, comparado con la parcela de Las Mercedes.

Comparación de la abundancia por finca. Se comparó la abundancia total de insectos por familia en ambas fincas (figura, 2). De manera general, la mayor abundancia se registró en la finca Las Mercedes. La mayor abundancia corresponde a las familias Apidae y Tachinidae y la menor abundancia a las familias Coreidae, Cerambycidae y Vespidae.

En el caso de los insectos de la familia Apidae, estos juegan un rol en el marango como polinizadores y productores de miel en época de floración, registrándose en gran número durante este período. Los insectos de la familia Tachinidae se registaron también en mayor número en la época de floración y fructificación, ya que estos insectos como las abejas y avispas son por lo general polinizadoras de flores y parasitoides de larvas de Lepidópteras, también se alimentan de mielecilla que producen las flores; algunas de ellas son parásitos de plaga. Entre los insectos que se encontraron en menor número, están los miembros de la familia Coreidae, principalmente chinches patas de hoja, cuya importancia económica es baja, y en marango casi nula. Los Coreidae se encontraron de manera esporádica y alimentándose del follaje. Los insectos de las familias Cerambycidae y Vespidae se encontraron en menor número en las trampas. Los Cerambycidae tienen un rol de plagas barrenadoras de árboles, pero en el marango, no se detectó este hábito, aunque de manera general las larvas de Cerambycidae pueden provocar daños en árboles de sombra, frutales y troncos recién cortados. Los Vespidae, son las avispas, que al igual que las abejas se observaron polinizando flores, alimentándose de mielecilla y en ocasiones se observaron algunas avispas depredando pequeñas larvas de mariposas.

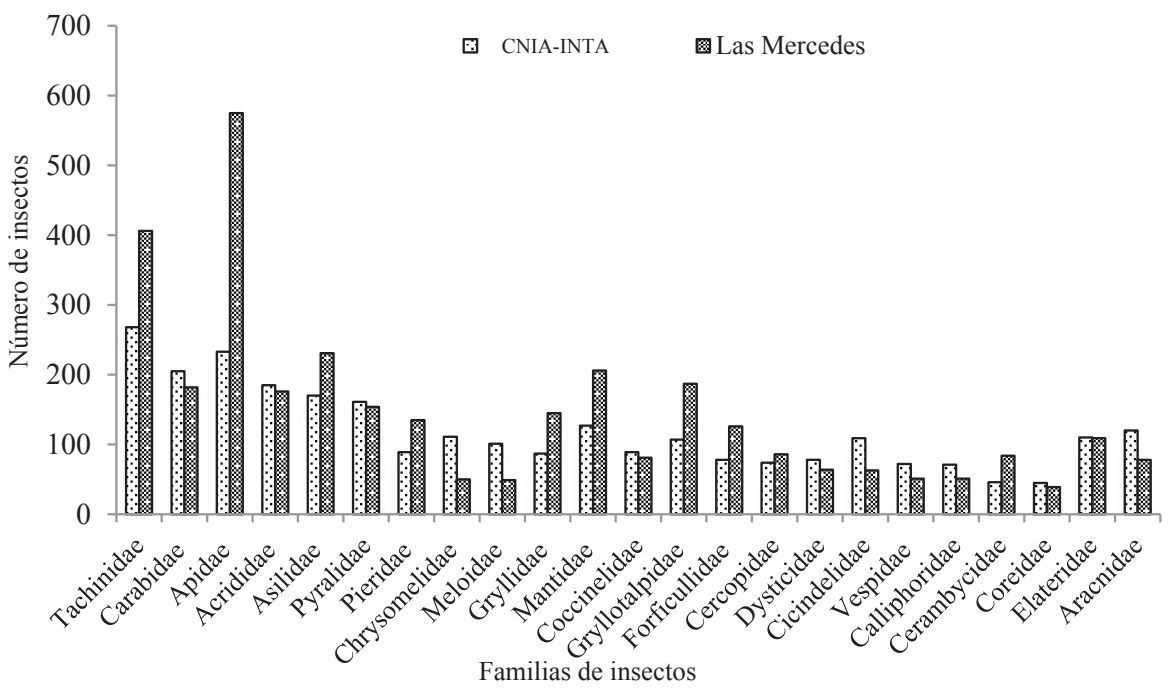

Riqueza. La riqueza total de especies fue de 156. En la parcela CNIA-INTA se registra mayor riqueza (88), mientras que en Las Mercedes se reportan 68. Consideramos que una de las posibles razones por la que se encontró mayor riqueza de especie en la parcela de marango del CNIA-INTA, es que ésta es una parcela donde el ciclo productivo del marango se da en su totalidad, encontrándose hojas, flores, frutos y semillas. Como el manejo de esta parcela está orientada a la obtención de material de propagación, por su fenología está llena de flores y vainas, lo que ocasiona una atracción de insectos con variedad de hábitos alimenticios.

Las plantas de la parcela CNIA-INTA son más desarrolladas que las de Las Mercedes, de mayor altura y más ramificadas, probablemente esto permita que haya mayor presencia de especies en estas plantas, agregado que cercano a esta parcela también están establecidos y se produce una variedad de cultivos como sorgo, maíz, frijoles, hortalizas, frutales y vegetales, lo que de alguna manera atraen más insectos y al emigrar de un cultivo a otro, tienen su paso por el cultivo del marango. 
Índice de diversidad (Shannon-Wiever) Se comparó el índice de diversidad de Shannon-Wiever de la finca CNIAINTA con el de Las Mercedes, resultando mayor índice promedio en la finca del CNIA-INTA (cuadro 2). En el caso de comparar la diversidad entre familias de insectos. para la finca del INTA, la diversidad encontrada fue entre 1.07 y 1.26, mientras que en la parcela de Las Mercedes, el índice de diversidad de las familias anduvo entre 1.05 y 1.35 .

Cuadro 2. Índice de diversidad Shannon-Wiever de las principales familias de insectos asociados al cultivo del marango en las fincas Las Mercedes y CNIA-INTA

\begin{tabular}{lcclcc}
\hline \multirow{2}{*}{ Familia } & \multicolumn{2}{c}{ Índice de Shannon-Weaver } & \multirow{2}{*}{ Familia } & \multicolumn{2}{c}{ Índice de Shannon-Weaver } \\
\cline { 2 - 3 } Tachinidae & CNIA-INTA & Las Mercedes & & CNIA-INTA & Las Mercedes \\
Carabidae & 1.26 & 1.29 & Coccinellidae & 1.14 & 1.18 \\
Apidae & 1.21 & 1.17 & Gryllotalpidae & 1.14 & 1.13 \\
Acrididae & 1.23 & 1.35 & Forficullidae & 1.10 & 1.10 \\
Asilidae & 1.19 & 1.20 & Cercopidae & 1.11 & 1.08 \\
Pyralidae & 1.18 & 1.15 & Dysticidae & 1.14 & 1.08 \\
Pieridae & 1.12 & 1.14 & Cicindellidae & 1.10 & 1.07 \\
Chrysomelidae & 1.14 & 1.07 & Vespidae & 1.10 & 1.07 \\
Meloidae & 1.13 & 1.06 & Calliphoridae & 1.07 & 1.10 \\
Gryllidae & 1.12 & 1.15 & Cerambycidae & 1.07 & 1.05 \\
Mantidae & 1.15 & 1.19 & Coreidae & 1.14 & 1.12 \\
\hline
\end{tabular}

\section{CONCLUSIONES}

Los principales ordenes de insectos encontrados asociados al cultivo del marango, fueron Orthóptera, Mantodea, Hemíptera, Coleóptera, Lepidóptera, Hymenóptera.

La mayor abundancia de insectos asociados al marango, fue encontrada en la finca Las Mercedes.

Las familias Apidae y Tachinidae, fueron las más abundantes en ambas parcelas, durante la época de floración y fructificación, estos insectos son por lo general polinizadoras y también se alimentan de mielecilla que producen las flores. La menor abundancia de insectos fueron los de las familias Coreidae, Cerambycidae y Vespidae. Estos fueron encontrados de manera esporádica en el marango alimentándose del follaje.
La mayor riqueza de insectos fue encontrada en la finca del CNIA-INTA, comparado con la finca Las Mercedes, esto se asocia al tipo de manejo que se da a esta parcela, donde el ciclo productivo del marango se da completo, encontrándose durante el ciclo hojas, flores, frutos y semillas, que atraen más insectos con variedad de hábitos alimenticios.

\section{AGRADECIMIENTO}

Los autores agradecen al Ing. Oswaldo Rodríguez por apoyar la identificación de los especímenes de insectos, al PROYECTO MARANGO de la UNA por financiar esta investigación, a la Universidad Nacional Agraria (UNA) por su apoyo en prestarnos la finca Las Mercedes donde establecimos el estudio y al INTA por facilitarnos su finca CNIA donde también se realizó parte de este estudio.

\section{REFERENCIAS BIBLIOGRÁFICAS}

Castellón, C; Gonzales, CH. 1996. Utilización del marango (Moringa oleífera) en la alimentación de novillos en crecimiento bajo régimen de estabulación, Tesis, Universidad Centroamericana. Facultad de Ciencias Agropecuarias. Managua, NI. 78 p.

D’Souza, J; Kulkarni, AR. 1990. Comparative studies on nutritive values of tender foliage of seedlings and mature plants of Moringa oleifera (Lamk.), Indian Journal of Nutrition and Dietetics. 27 (7): 205-212.

Duke, 2003. La biología de termitas subterráneas del este de los Estados Unidos. Universidad de Georgia, USA. p 9.

FAO. (Organización de las Naciones Unidas para la Alimentación y la Agricultura, IT). 2003 Estrategia regional para sanidad y manejo forestal en América Central, San José CR. 27 p.

Jiménez-Martínez, ES. 2009. Manejo integrado de plagas. Universidad Nacional Agraria, Managua, NI. 108 p.

Johnson, BC. 2005. Health effects of Moringa oleifera: a promising adjunct for balanced nutrition and better health. KOS Health Publications, 2 (6): 1-5.

Makkar, HPS; Becker, K. 1997. Nutrients and antiquality factor in different morphological parts of Moringa oleifera tree. Journal of agricultural science (Camb) 128: 311-322.

Martínez, L.; Reyes S, N; Rocha M, L. 2011. Plan nacional de fomento del cultivo y utilización de marango (Moringa oleifera) en Nicaragua, Universidad Nacional Agraria. 22 p.

Nunes Zuffo, C; Dávila Arce, ML. 2004. Taxonomía de las principales familias y subfamilias de insectos de interés agrícolas en Nicaragua. UCATSE (Universidad Católica Agropecuaria del Trópico Seco), Estelí, NI. 164 p. 


\section{REECURSOS NATUVRALES}

Nikolaus, F.; Mayorga, L.; Vásquez, W. 1998. Proyecto biomasa. Managua, NI. (en línea). Consultado: 24 de oct. 2012 Disponible en: biomasa@ibw.com.ni

Rajangam, J; Azahakia, R; Manavalan, S; Thangaraj, T; Vijayakumar, A. 2001. The miracle tree / the multiple attributes of moringa. Dakar: CWS; 2001. Muthukrishan N. Status of production and utilization of moringa in Southern India; p. 45-47.

Sáenz, M; De La Llana, A. 1990. Entomología sistemática. Universidad Nacional Agraria. Managua, NI. 136 p.

SAS Institute, 2003. University of Nebraska. Cary, NC, USA.V.91.

Shannon, C.E., Weaver, W. 1949. The mathematical theory of communication, Urbana, Illinois. University of Illinois Press, 117 p.

UNA (Universidad Nacional Agraria). 2004. Marango: Cultivo y utilización en la alimentación animal. Guía Técnica ํ․ 5. Managua, NI. 53 p. 